\title{
Electrooptic and Electroabsorptive Modulation Properties in Interdiffusion-Modified AlGaAs-GaAs Quantum Wells
}

\author{
E. Herbert Li, Senior Member, IEEE, and Wallace C. H. Choy, Student Member, IEEE
}

\begin{abstract}
The electric field induced refractive index change and absorption coefficient change in TE polarization are analyzed at room temperature for several interdinusion modifed Alo.3 Gao.7 As-GaAs quantum-well structures. The results show that for the small and medium interdimusion lengths with fields of 100 and $50 \mathrm{kV} / \mathrm{cm}$, respectively, improved chirplang and elec. troabsorption can be obtained. Further, in a selected set of interdifusion lengths and fields, the material can be used for an electroabsorption modulator with reduced chinping in a wide range of operation wavelengths $(758-874 \mathrm{~mm})$.
\end{abstract}

$\mathbf{T}$ HERE HAS been a considerable interest in the electrooptic effects in semiconductor quantum wells (QW's) for their improved performance in various optoelectronic devices [1]. This is because of a large change in the electric field induced refractive index variation $(\Delta n / n \approx 1 \%)$ due to the quantum confined Stark effect (QCSE) in the QW's around the excitonic absorption edge as compared to those in conventional materials, such as bulk III-V semiconductors $(\Delta n / n \approx$ $0.01 \%)$ and $\operatorname{LiNbO3}(\Delta n / n \approx 0.1 \%)$. Recently the technique of using thermal annealing to produce diffusion modified non-square QW's (DFQW's) has received much attention for the monolithic integration of devices, such as waveguides, modulators, and lasers, in optoelectronic integrated circuits [2]. The DFQW structures are also attractive in its own merit due to their improved performance in waveguide-modulators [3] and lasers [4]. However, there is a need to study the electrooptic effect in such structures since a large $\Delta n$ is required for directional couplers and it is important to have a small $\Delta n$ in high-speed absorption modulators due to chirping. This is the first theoretical study on the electrooptic and electroabsorptive effects and their composition effect in the DFQW.

In this letter, both the electroabsorption and electrooptic effects are studied theoretically by analyzing the electric field induced absorption and refractive index changes in DFQW at room temperature. We will first discuss the $\Delta n$ of $\mathrm{DFQW}$ with various extent of interdiffusion $L_{d}$ at two applied electric fields and its contribution on phase modulator, this is followed by a study on the chirping of DFQW for applications in high-intensity/speed modulators. Finally, several structures of DFQW for electroabsorption and electrooptical modulation

Manuscript received December 28, 1994; revised March 31, 1995. This work was supported in part by a HKU-CRCG Grant.

The authors are with the University of Hong Kong, Department of Electrical and Electronic Engineering, Hong Kong.

IEEE Log Number 9412830 will be discussed. The as-grown $\mathrm{QW}$ to be analyzed consists of a $100-\AA$ width $\mathrm{GaAs}$ well and thick $\mathrm{Al}_{0.3} \mathrm{Ga}_{0.7} \mathrm{As}-\mathrm{GaAs}$ barriers. The DFQW is modeled by an error function and the extent of interdiffusion is characterized by a diffusion length $L_{d}=(D t)^{1 / 2}$, where $D$ and $t$ are the diffusion coefficient and annealing time respectively. To calculate the subband wavefunctions and dispersion including the effect of valenceband mixing, the $k \cdot p$ method is used; the detail of these calculations and the AlGaAs material parameters used are presented in [5]. The $\Delta n$ considered here is associated with the QCSE and the linear electrooptic effect is neglected since it is small; in accordance to the sum rules derived for QW's [6] the $\Delta n$ can be calculated using the Kramers-Krönig relation.

Fig. 1 shows the TE polarized $n$ in several DFQW structures $\left(0<L_{d}<40 \AA\right)$ for two cases of electric fields, (a) $F=$ $30 \mathrm{kV} / \mathrm{cm}$ and (b) $F=50 \mathrm{kV} / \mathrm{cm}$. The corresponding zero field absorption coefficient is also shown in Fig. 1(c) as a reference, it can be seen that both the heavy- and lighthole excitons appear for all cases except at $L_{d}=40 \AA$. At wavelengths above and below the two $1 S$ exciton peaks, $\Delta n$ is positive and with a two $W$ wave-like (this is defined as the shape of the third derivative of a Gaussian function) dispersion situated in between these peaks. This is thought to be due to the two excitons related $\Delta \alpha$, which behave as a two- $N$ wave-like (first derivative) dispersion before the Kramers-Krönig transformation is performed. It is apparent from Fig. 1(a) and (b) that the maximum $n / n$ is negative and varies between -0.03 to -0.015 for the $F=30 \mathrm{kV} / \mathrm{cm}$ case, which corresponds to a $\Delta n / n$ of 0.8 and $0.4 \%$, respectively. The case of $F=50 \mathrm{kV} / \mathrm{cm}$ is similar in trend to the previous case with the percentage change in $\Delta n / n$ varying between 0.9 and $1.3 \%$. These values are in good agreement with both experimental [7] and theoretical [8] results obtained from the as-grown square QW's. An interesting feature to be observed in both cases is that $\Delta n_{\max }$ decreases initially with increasing $L_{d}$, and then recovers to about the same strength as $L_{d}$ reaches 40 A; however, the spectra become much broader and for the case of $F=50 \mathrm{kV} / \mathrm{cm}$ the sum rule is being somewhat violated. On the other hand the $\Delta n_{\max }$ variation for different DFQW's under the $F=50 \mathrm{kV} / \mathrm{cm}$ is much reduced and may have potential applications in wide-bandwidth electrooptic devices.

The phase modulation due to nmax can be calculated by considering an ideal situation for the phase difference per unit length, $\Delta \phi=2 \pi \Delta n / \lambda$, in a waveguide-modulator type of 
(a)

(b)

(c)

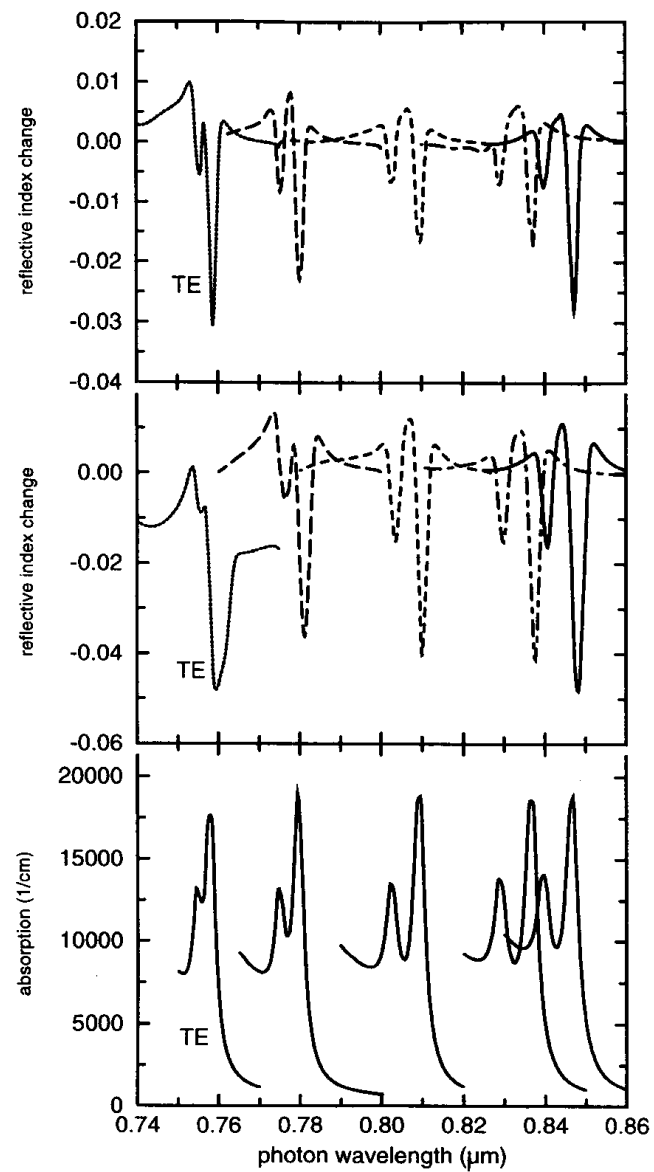

Fig. 1. The TE polarized light electrooptic spectra, $\Delta n$, of $100-\AA$-wide $\mathrm{Al}_{0.3} \mathrm{Ga}_{0.7} \mathrm{As}-\mathrm{GaAs} \mathrm{QW}$, at electric field of (a) $F=30 \mathrm{kV} / \mathrm{cm}$ and (b) $50 \mathrm{kV} / \mathrm{cm}$ for different DFQW structures: $L_{d}=0 \AA$ (solid), $10 \AA$ (dot-dash), $20 \AA$ (dash), $30 \AA$ (long dash), and $40 \AA$ (dot). Their respective absorption coefficient at zero-field is also shown in (c) as a reference.

structure. It should be noted that the values obtained may only be used as an indicator although a confinement factor of 0.0025 per QW has already been taken into consideration for scaling purposes. For the $F=30 \mathrm{kV} / \mathrm{cm}$ case, $\Delta \phi\left(L_{d}\right.$ in $)=$ 31.95 (0), 17.52 (10), 18.06 (20), 25.96 (30), 35.53 (40) $\% / \mathrm{mm}$, and for the $F=50 \mathrm{kV} / \mathrm{cm}$ case, $\Delta \phi=53.07(0)$, 45.65 (10), 44.45 (20), 41.38 (30), $47.37(40) \% / m m$. These results indicate a distinctive $\Delta \phi$ in the former case that can be served in a multiplexer type of application while the latter case could be used as a wide-bandwidth directional coupler modulator/detector.

In the interest of high-speed intensity modulators, the effect of DFQW on the chirping problem of electroabsorptive modulation is important since this will affect the frequency (wavelength) broadening of the output intensity spectrum of the device. The degree of chirping can be indicated by a linewidth enhancement factor $\beta_{\bmod }$ [7], which is due to an electric field applied in the modulator as in the case of charge carrier injected into the laser device. The relation is defined as:

$$
\beta_{\mathrm{mod}}=\Delta n(\lambda) / \Delta k(\lambda)
$$

where $\Delta k$ is the change of extinction coefficient that corresponds to the imaginary part of the complex refractive index. Physically, a change in the absorption coefficient implies a change in the extinction coefficient since they are related by $\Delta k=\lambda \Delta \alpha /(4 \pi)$, and through Kramers-Krönig relations, this change in $\Delta k$ leads to a change in $\Delta n$. So, as a whole, the electrooptic effect is in principle related to electroabsorption while the factor $\beta_{\text {mod }}$ can be considered as a ratio between them.

The chirping effect will now be analyzed in terms of $\beta_{\text {mod }}$ with the same DFQW structures analyzed earlier. In order to facilitate discussion, the spectra of $\beta_{\text {mod }}, \Delta n$ with $\Delta k$ together, and $\alpha(F=0,100 \mathrm{kV} / \mathrm{cm})$ are shown in Fig. 2(a)-(c), respectively, for $L_{d}=30 \AA$ and $F=100 \mathrm{kV} / \mathrm{cm}$. An apparent feature shows that there are three dispersible singularities in the $\beta_{\text {mod }}$ spectrum, which implies that the spectrum of $\Delta k$ has crossed the zero point $(\Delta k=\Delta \alpha=0)$ three times. At these points, $\beta_{\text {mod }}$ should theoretically tend to $\infty$ since the material mainly contributes to electrooptic effect without any change in electroabsorption. The reason the singularities in the present calculation do not tend to infinity is due to finesse. So far infinite chirping (wavelength chirp to infinity) have not yet been observed, however, it is expected that $\beta_{\text {mod }}$ is large but finite rather than infinite in magnitude. On the other hand, between any two consecutive singular points $\beta_{\text {mod }}$ must crosses zero. This denotes that $\Delta n=0$ while $\Delta \alpha$ remains finite and non-zero; the material now attributes mainly to electroabsorption with no contribution to the electrooptic effect. The sign of $\beta_{\text {mod }}$ depends on that of $\Delta n$ and $\Delta \alpha$, and there are two possible cases to be considered as follows. First, for $\beta_{\text {mod }}>0$, which occurs in certain wavelength ranges, both parameters $(\Delta n, \Delta \alpha)$ are negative. When $\Delta n$ is negative, the phase modulation is negative, and since $\Delta \alpha$ is also negative, it improves the transparency so that more light can be transmitted out. Therefore, this implies a relatively larger intensity of the output light with a phase reduction. Second, for $\beta_{\text {mod }}<0$, $\Delta n$ and $\Delta \alpha$ are in opposite signs with $\Delta n<0$ and $\Delta \alpha>0$, this results in a reduction of the output light intensity with a phase increment. In addition the sign of $\beta_{\text {mod }}$ changes as the zero points are crossed, since $\Delta \alpha$ changes sign while $\Delta n$ keeps the same sign. An interesting feature to note at around $\Delta n_{\min }$ when the slope of $\Delta n$ changes sign from negative to positive in the range from $\lambda=780-782.5 \mathrm{~nm}$, respectively, the light signal with an increasing positive slope of $n$ will blue-shift in energy and that with a decreasing negative slope of $\Delta n$ will red-shift in energy. Therefore an input light pulse, centered at the above minimum point of $\Delta n$ and spread within the respective wavelength range will be compressed, thus this can be used in pulse compression applications. Fig. 2(c) shows the residence loss as a reference; it is also seen that the heavyand light-hole exciton peaks mix together and undergo a red shift when $F=100 \mathrm{kV} / \mathrm{cm}$. It brings a large $\Delta \alpha$ at $779.5 \mathrm{~nm}$ for application in light intensity modulations.

In order to have a high-performance modulator based on electroabsorption, only a selected range of wavelengths covering $\Delta \alpha_{\max }$ is taken into consideration. Table I shows the wavelength positions of the chirping singularities for several cases of $L_{d}$ and at two fields. The operation wavelength, $\lambda_{\text {op }}$, 
(a)

(b)

(c)

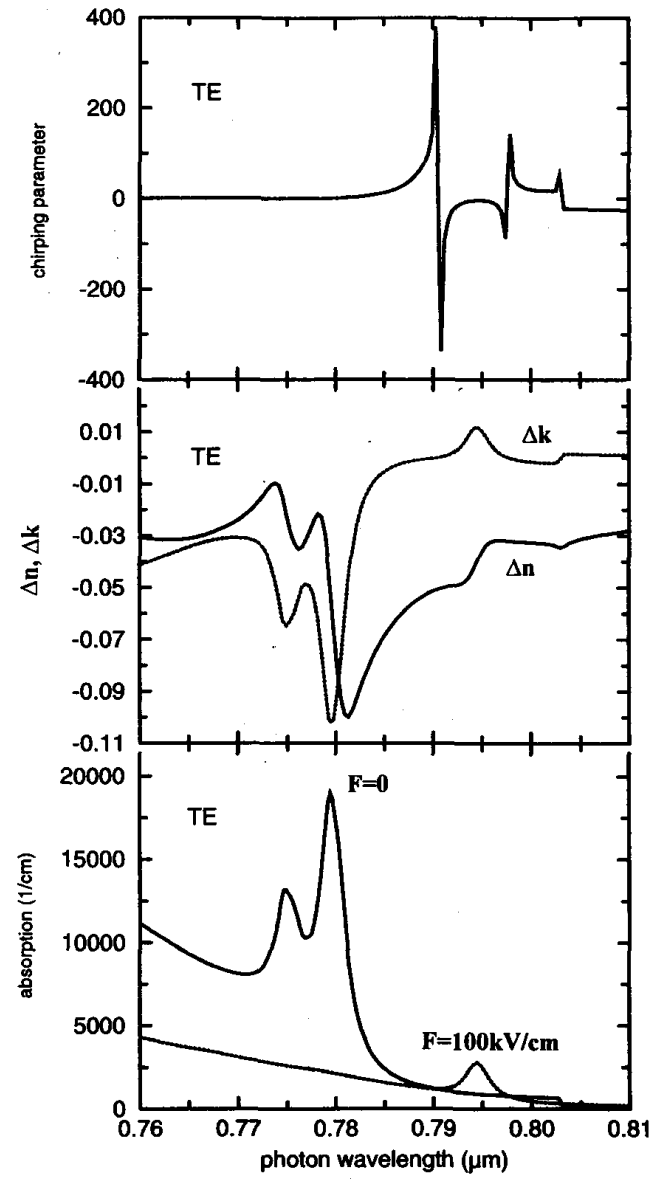

Fig. 2. (a) The chirping coefficient $\beta_{\text {mod }}$ for $\mathrm{Al}_{0.3} \mathrm{Ga}_{0.7} \mathrm{As} / \mathrm{GaAs}$ with $L_{z}=100 \AA, L_{d}=30 \AA$ and $F=100 \mathrm{kV} / \mathrm{cm}$. Three dispersible singularities in the spectrum between 785 and $805 \mathrm{~nm}$. (b) $\Delta n$ (solid line) and $\Delta k$ (dot line) for the same structure as in (a). (c) The absorption spectra of the case $L_{d}=30 \AA$ with $F=0$ (solid line) and $F=100 \mathrm{kV} / \mathrm{cm}$ (dot line).

is taken at the respective exciton absorption peaks with field for $L_{d}=0-20 \AA$ and without field for $L_{d}=30$ and $40 \AA$, which are far enough from the nearest chirping singularity. The modulation OFF-state is taken at the unbias state $(F=0)$ for $L_{d}=0-20 \AA$ and at the bias state $(F \neq 0)$ for $L_{d}=$ 30 and $40 \AA$. As $L_{d}$ increase, the wavelength of $\Delta \alpha_{\max }$ as well as the chirping singularities undergo a blue shift because the bandgap energy of the DFQW increase [5]. In the case of $F=100 \mathrm{kV} / \mathrm{cm}$, both $\Delta \alpha\left(\lambda_{o p}\right)$ and $\beta_{\bmod }\left(\lambda_{o p}\right)$ fluctuate between $L_{d}=0$ and $30 \AA$. As a whole $\Delta \alpha\left(\lambda_{o p}\right)$ always increases with respect to the square $\mathrm{QW}$ case. In fact, $\Delta \alpha\left(\lambda_{o p}\right)$ increases by one and half times for the case of $L_{d}=10 \AA$ and by two fold for the case of $L_{d}=30 \AA$ while the $\left|\beta_{\bmod }\left(\lambda_{o p}\right)\right|$ is halved at $L_{d}=10 \AA$, with respect to those of $L_{d}=0$. Therefore an improved modulation, doubled $\Delta \alpha\left(\lambda_{o p}\right)$ and halved $\left|\beta_{\bmod }\left(\lambda_{o p}\right)\right|$, can be obtained with an increased but acceptable residence loss (1163.5 900)/900 = $29 \%$ for the case of $L_{d}=10 \AA$. Above all, it is worth noting that when the interdiffusion further increases to $L_{d}=$ $40 \AA$, only a halved applied field $(50 \mathrm{kV} / \mathrm{cm})$ is required to produce a $\Delta \alpha_{o p}$ similar to that in the case of $L_{d}=30 \AA$ (but
TABLE I

The WaVELENGTH (nm) OF THE SingulartTy of $\boldsymbol{\beta}_{\text {mod }}$, I.E.,

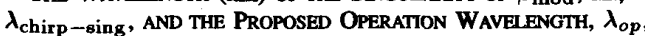
AND ITS CORRESPONDING $\alpha(F), \alpha(F=0), \Delta \alpha$, AND $\beta_{\text {mod }}$ ARE SHOWN POR SEVERAL $L_{d}$ 's AND $F=100,50 \mathrm{kV} / \mathrm{cm}$.

\begin{tabular}{|l|l|l|l|l|l|l|}
\hline $\mathrm{L}_{\mathrm{d}}$ & $\AA$ & 0 & 10 & 20 & 30 & 40 \\
\hline operation state & & unbias & unbias & unbias & bias & bias \\
\hline $\mathrm{F}$ & $\mathrm{kV} / \mathrm{cm}$ & 100 & 100 & 100 & 100 & 50 \\
\hline$\lambda_{\text {chirp-sing }}$ & $\mathrm{nm}$ & 873.5 & 862.0 & 828.0 & 790.5 & 761.5 \\
\hline$\lambda_{\text {op }}$ & $\mathrm{nm}$ & 862.1 & 848.0 & 819.5 & 779.5 & 757.8 \\
\hline$\alpha_{\mathrm{F}}\left(\lambda=\lambda_{\text {op }}\right)$ & $1 / \mathrm{cm}$ & 10540 & 16246 & 11288 & 2174 & 2286 \\
\hline$\alpha_{\mathrm{f}=0}\left(\lambda=\lambda_{\text {op }}\right)$ & $1 / \mathrm{cm}$ & 900 & 1164 & 1268 & 18907 & 17551 \\
\hline$\Delta \alpha\left(\lambda=\lambda_{\text {op }}\right)$ & $1 / \mathrm{cm}$ & 9640 & 15083 & 10020 & 16733 & 15265 \\
\hline$\beta_{\text {mod }}\left(\lambda=\lambda_{\text {op }}\right)$ & - & -0.815 & -0.402 & -0.687 & 0.620 & 0.520 \\
\hline
\end{tabular}

still double to that of square $\mathrm{QW}$ ). This reduces the voltage swing by half to operate the device. The DFQW may also be applied as a tunable intensity-type modulator with wavelengths ranged from $758-874 \mathrm{~nm}(\Delta \lambda=116 \mathrm{~nm})$ by varying the interdiffusion (different $L_{d}$ 's).

In conclusion, we have analyzed the electrooptic effect in DFQW's where results indicate a larger variation in $\Delta n_{\max }$ for a smaller field while a stronger and an almost constant $\Delta n_{\max }$ is found for the larger field case. The phase modulation properties due to these effects in the DFQW's suggest some types of multi-wavelengths application can be realized by means of fabricating planar integrated multi-section modulators/detectors on a single substrate through the technique of selective area interdiffusion. However care must be taken in the DFQW design, such to choose the best interdiffusion length and electric field. For example at $L_{d}=10$ and $40 \AA$ with applied field $F=100 \mathrm{kV} / \mathrm{cm}$ and $F=50 \mathrm{kV} / \mathrm{cm}$, respectively, the DFQW's provide large electroabsorption and less chirping in a selected range of wavelengths. These make the DFQW's become competitive as an electroabsorption modulator. Finally, the performance of these DFQW devices may be further improved by optimizing the DFQW structure and together with the use of strained layered QW materials.

\section{REFERENCES}

[1] S. K. Han, R. V. Ramaswamy, W. Q. Li, and P. K. Bhattacharya, "Efficient electro-optic modulator in InGaAlAs/InP optical waveguides," IEEE Photon. Technol Lett. vol. 5, no. 1, pp. 46-49, 1993.

[2] O. Wada, A. Furuya, and M. Makiuchi, "Planar, compatible OEIC's based on multiquantum well structures," IEEE Photon. Technol. Lett., vol. 1, no. 1, p. 16-18, 1989.

[3] E. H. Li and B. L. Weiss, "Enhanced electroabsorption in disordered AlGaAs/GaAs quantum wells," IEEE Photon. Technol. Lett., vol. 4, no. 4 , pp. $445-448,1993$.

[4] E. H. Li and K. S. Chan, "Laser gain and current density in a disordered AlGaAs/GaAs quantum well," Electron. Lett., vol. 29, pp. 1233-1234, 1993.

[5] E. H. Li, B. L. Weiss, and K. S. Chan, "Effect of interdiffusion on the subband structure in an AlGaAs/GaAs single quantum well structure," Phys. Rev. B., vol. 46, pp. 15181-15192, 1992.

[6] D. A. B. Miller, J. S. Weiner, and D. S. Chemla, "Electric-field dependence of linear optical properties in quantum well structures: Waveguide electroabsorption and sum rules," IEEE J. Quantum Electrom, vol. QE22, pp. $1816-1830,1986$.

[7] J. S. Weiner, D. A. B. Miller, and D. S. Chemla, "Quadratic electrooptic effect due to the quantum-confined Stark effect in quantum wells," Appl. Phys. Lett., vol. 50, pp. 842-844, 1987.

[8] T. Hiroshima, "Electric field induced refractive index changes in GaAs-AlGaAs quantum wells," Appl. Phys. Lett., vol. 50, pp. 968-970, 1987. 